\title{
ERLÄUTERUNGEN ZU DEN KARTEN TYPISCHER WETTERLAGEN IM SCHWEIZERISCHEN MITTELSCHULATLAS (Seite 77)
}

\section{Von MaX SchÜEpp}

Der neue Mittelschulatlas bringt ein vergrößertes wetterkundliches Material, indem sechs Grundtypen von Wetterkarten an die Stelle einer einzelnen Föhnlage treten. Ermöglicht wurde diese Erweiterung durch das große Verständnis von Herrn Prof. Imhof, der die Mitarbeiter für das Projekt gewinnen und den nötigen Platz im Atlas freimachen konnte. Für seine vielfachen Bemühungen sei ihm auch an dieser Stelle herzlicher Dank ausgesprochen.

Der Witterungscharakter in einem bestimmten Gebiet wird im wesentlichen durch zwei Faktoren bestimmt:

1. Durch die Advektion, d. h. die horizontale Luftzufuhr;

2. Durch die Konvektion, d. h. die vertikalen Umlagerungen in der Luftmasse.

Die Advektion kann je nach der Lage der vorherrschenden Hoch- und Tiefdruckgebiete aus verschiedenen Himmelsrichtungen erfolgen. Wir können daher vier Hauptklassen unterscheiden, je nachdem die Luft aus S, W, N oder E heranströmt.

Bei der Konvektion unterscheiden wir zwischen aufsteigender und absteigender Bewegung (zyklonale und antizyklonale Luftdruckverhältnisse).

Wir erhalten daher sechs Grundtypen, aus deren Kombination sich die einzelnen, stets wechselnden Wettersituationen zusammensetzen. Zwar kommen die verschiedenen Grundtypen entweder rein oder modifiziert in allen Jahreszeiten vor, aber im langjährigen Durchschnitt zeigen sich doch jahreszeitliche Häufungen. Die vier Advektionstypen sind besonders für die kältere Jahreszeit charakteristisch, weil dann auf der Nordhalbkugel die Temperatur- und Luftdruckunterschiede zwischen polaren und subtropischen Gegenden besonders groß sind. Dagegen fehlt in unserem Gebiet im Winter die starke Sonneneinstrahlung, die im Frühling und Sommer die aufsteigenden Konvektionsströmungen begünstigt. Wir finden dann nur den zweiten, negativen Konvektionstypus, das antizyklonale Absinken im Zentrum eines Hochs und auch diesen Typus meist nur für kurze Zeit. Länger andauernd sind die antizyklonalen Schönwetterlagen oft im Herbst und Vorwinter sowie im Vorfrühling.

Wir haben die Hauptwettertypen mit den im schweizerischen Gebiet gebräuchlichen Namen bezeichnet. Wichtig ist aber nicht die lokale Wettererscheinung, sondern die allgemeine Luftmassenzufuhr, die uns je nachdem aus dem Süden warme Tropikluft, aus dem Westen feuchte, aus dem Osten dagegen trockene Polarluft oder aus dem Norden feuchte Polar- oder Arktikluft heranbringt.

Wir betrachten also nicht nur das Luftdruckbild, sondern auch die gegenseitige Lage der verschiedenen Luftmassen. Daher wurden durch die Farben nicht die Hochund Tiefdruckzentren, sondern Warm- und Kaltluftgebiete voneinander getrennt. Die Grenzlinien zwischen diesen Gebieten bilden die sogenannten Fronten. Als Warmfront werden Teile dieser Grenze bezeichnet, bei denen die Warmluft sich über die vorgelagerte Kaltluft schiebt, während sich bei der Kaltfront die Kaltluft unter die vorgelagerte Warmluft drängt. Bei der sogenannten Okklusion hat die Kaltfront die davorliegende Warmfront eingeholt und die Warmluft vom Boden abgehoben, so daß sie nur noch in den höheren Luftschichten vorhanden ist (siehe Westwindwetterlage). Die Fronten bewegen sich in Richtung der Schuppen, die bei der Symboldarstellung angegeben sind. Die Warmfrontschuppen sind in unseren Beispielen im Atlas vom Zeichner eckig entworfen worden. Nach internationaler Vereinbarung werden sie 
abgerundet gezeichnet. Die Kaltfront wird durch Zacken, die Okklusion durch gemischte Schuppen dargestellt.

Die Fronten geben sich vor allem durch charakteristische Bewölkungs- und Niederschlagsgebiete sowie durch Luftdruckänderungen in der Umgebung auf der Wetterkarte zu erkennen. Dagegen ist die Temperaturdifferenz in den bodennahen Luftschichten oft nicht bedeutend, besonders in den Karten des Vormittagstermins $07 \mathrm{Uhr}$, da infolge der nächtlichen Ausstrahlung lokale Abkühlungen in Bodennähe auftreten (Bodeninversionen). Nur im Beispiel der Gewitterlage zeigt sich ein scharfer Temperaturgegensatz von etwa $10^{\circ} \mathrm{zwischen} \mathrm{Kalt-und} \mathrm{Warmluft.} \mathrm{In} \mathrm{diesem} \mathrm{Beispiel} \mathrm{eilt} \mathrm{die} \mathrm{kühlere}$ Luft in den höheren Atmosphärenschichten über Frankreich und Belgien der unteren Kaltluft voraus. (Die Front ist dort für die höheren, voreilenden Schichten eingezeichnet.) In den anderen Fällen lagert die kalte, schwere Luft vor allem in den unteren Luftschichten. Darüber kann sich in der Höhe Warmluft befinden (z. B. in Okklusionsgebieten und in den nordöstlichen Teilen von warmen Antizyklonen). Diese Gebiete sind mit violetter Farbe bezeichnet. Bevor wir zur Besprechung unserer sechs Haupttypen übergehen, ein kurzer Blick auf die Zeichenerklärung:

Wir haben überall die in den offiziellen Wetterkarten verwendeten und allgemein gebräuchlichen Symbole verwendet und bei den einzelnen Stationsangaben in der Wetterkarte Wind (Richtung und Stärke, mit Pfeilen), Temperatur (in Grad Celsius in Zahlen) sowie Bewölkungsgrad und Wetter (mit Symbolen) dargestellt. Die Isobaren sind in dem heute in der Meteorologie allgemein verwendeten Millibarmaß angegeben, das in das internationale CGS-Maßsystem eingepaßt ist, während der Millimeter der Quecksilbersäule eine willkürliche Einheit darstellt. Die Umrechnung von Millimeter $(\mathrm{mm})$ in Millibar (mb) und umgekehrt ist sehr einfach: $1 \mathrm{mb}=3 / 4 \mathrm{~mm}$ oder $1000 \mathrm{mb}$ $=750 \mathrm{~mm}$. (Mittlerer Luftdruck auf Meeresniveau in unserer Gegend zirka $1015 \mathrm{mb}$.) Niederschlagsgebiete sind schraffiert.

Um ausführlichere Angaben über das Wetter in der Schweiz zu haben, ist unter den einzelnenWetterkarten jeweils eineAuswahl von Wetterbeobachtungen einzelnerStationen angegeben, die Zahlenangaben für charakteristische Wetterelemente enthalten. Wir werden nachfolgend bei der Besprechung der einzelnen Wetterlagen näher darauf eingehen.

1. Die Föhnlage: Die den Föhn erzeugenden Tiefdruckgebiete entstehen 'normalerweise auf dem westlichen Atlantik südöstlich von Neufundland. Wir können daher ihre ganze Lebensgeschichte auf den Atlantikkarten (z. B. im tägtichen Wetterbulletin der Meteorologischen Zentralanstalt links unten) verfolgen. Wenn diese Depressionen nicht, wie in den meisten Fällen, gegen Nordeuropa ziehen, sondern einen südlicheren Kurs einschlagen, erzeugen sie beim Eintreffen an der europäischen Westküste Föhnlage. Wenn das Tief, wie in unserem Beispiel vom 25. 10. 1937, bis zu den britischen Inseln gelangt ist, hat es sich meist schon stark verwirbelt (okkludiert) und verlagert sich nicht mehr rasch. Es wird stationär, ein sogenanntes Zentraltief. Bei dieser Lage kann sich ein Süd-Nord-Strom von (sub-)tropischer Warmluft aus dem Mittelmeer und von Afrika her nach Norden entfalten und die Alpen überqueren. In vereinzelten Fällen ist die Strömung so stark, daß sie Saharastaub mit sich führt. Wir haben Föhnlage. Die außergewöhnliche Wärme und Trockenheit in den Föhntälern ist jedoch nicht nur auf den Ursprungsort der Luftmassen zurückzuführen, sondern vor allem auf die Wirkung des Gebirges. Die sechs angeführten .Terminbeobachtungen von schweizerischen Stationen auf einem Nord-Süd-Schnitt über die Alpen bieten die Möglichkeit, die typischen Erscheinungen des Föhns durch den Schüler selbst erarbeiten zu lassen'1.

Im aufsteigenden Teil der Föhnströmung sehen wir eine Temperaturabnahme von $11^{\circ}$ für den Höhenunterschied von $1800 \mathrm{~m}$ von Lugano bis zum Gotthard, also wenig

1 Bei der Temperatur des Gotthard fehlt infolge eines Druckfehlers das Minuszeichen. Sie muß von $2^{\circ}$ auf $-2^{\circ}$ verbessert werden. 
mehr als $1 / 2^{\circ}$ pro $100 \mathrm{~m}$. Infolge der freiwerdenden Kondensationswärme bei der Niederschlagsbildung kühlt sich die Luft beim Aufsteigen am Alpensüdfuß nur langsam ab. Die kräftige Föhnströmung, die wir auf dem Gotthard, dem Rigi und besonders in Altdorf beobachten, findet sich im Tessin nur in den höheren Luftschichten (oberhalb $1500-2000 \mathrm{~m}$ ). In den unteren Luftschichten bildet sich ein schwacher Luvwirbel, Lugano hat daher leichten Nordwind.

Nördlich des Alpenkammes, wo der Föhn in die Täler hinabstürzt, erwärmt er sich um $1^{\circ}$ pro $100 \mathrm{~m}$ (Temperaturdifferenz Altdorf-Gotthard $17^{\circ}$ für $1700 \mathrm{~m}$ Höhenunterschied). Im Föhntal finden wir klaren Himmel und hohe Temperatur sowie geringe Feuchtigkeit $\left(15^{\circ}\right.$ und $31 \%$ ). Im Mittelland dagegen liegt eine flache Kaltluftschicht von einigen hundert Metern Mächtigkeit, über welche die Föhnströmung hinweggleitet (z. B. in Luzern $5^{\circ}$ und $96 \%$ !). Der Luftdruckunterschied zwischen Luzern und Lugano beträgt etwa $9 \mathrm{mb}$. In den Föhntälern, in der leichten Warmluft, treten lokale Druckminima auf (vgl. die auf Meeresniveau reduzierten Drucke von Altdorf und Luzern). In den nördlichen Teilen der Schweiz, wo der Föhneinfluß abnimmt, herrscht bewölktes Wetter (Basel), während im Alpenvorland nur lokale Wolkenfelder, besonders über Bergkämmen auftreten («Wolkenfische», die Wellenbildungen in der Atmosphäre anzeigen).

Die Wetterkarte vom Abend des 25. Oktober 1937 zeigt uns die voll entwickelte Föhnlage mit dem charakteristischen «Föhnknie», dem Keil hohen Druckes, der sich in der durch den Alpenwall geschützten Poebene erhalten konnte, während der Druck im Westen gefallen ist. An der Grenze zwischen Warm- und Kaltluft, an der sogenannten Polarfront, entwickelt sich über Nordwestspanien ein neuer Tiefdruckkern, der bis zum nächsten Tag schon stärker sein wird als das alte Zentrum über England. Dieser Vorgang kann sich mehrere Male wiederholen, meist jedesmal etwas östlicher als vorher. Die Teiltiefe entstehen und vergehen rasch. Gelegentlich dringt ein Schwall Polarluft bis nach Mitteleuropa vor, ist aber zu wenig mächtig, um die Warmluft völlig zu verdrängen. Der Föhn «kämpft», oft mehrere Tage lang. Zum Schluß erfolgt entweder ein langsames Abflauen der Druckgegensätze oder (wenn die Kaltluftzufuhr von Westen her genügend stark ist) der Föhnzusammenbruch. Die Polarluft überflutet in diesem Falle Mitteleuropa. Der Druck steigt auf der Alpennordseite. Die Föhnlage geht in Westwindwetter oder sogar (bei raschem und starkem Anstieg über Westeuropa) in eine Staulage über.

2. Das Westwindwetter stellt in unserer Gegend den häufigsten Wettertypus dar. Es entspricht besonders im Vorfrühling und Spätherbst annähernd der durchschnittlichen (klimatologischen) Druckverteilung. Der subtropische Hochdruckgürtel (Passatzirkulation Äquator-Subtropen) und die Tiefdruckzone der gemäßigten Breiten (Polarfrontgebiet, d. h. Grenzgebiet zwischen [sub-]tropischer Warmluft und polarer Kaltluft) verursachen in unseren Breiten normalerweise eine Westströmung (vgl. die Klimakarten, wobei zu beachten ist, $\mathrm{da} ß$ auf der Nordhalbkugel die allgemeine Zirkulation im Sommer und Winter über Asien und Nordamerika durch die thermischen Tiefbzw. Hochdruckgebiete gestört ist). Diese Temperatureinflüsse überlagern sich der Grundströmung und verändern sie über den Kontinenten sehr stark (Monsun usw.). Der subtropische Hochdruckgürtel kann sich besonders ungestört über dem Ozean entwickeln: Azorenhoch, zeitweise in Richtung Spanien-Mitteleuropa vorstoßend. Die Tiefdrucktätigkeit ist ebenfalls am stärksten auf dem Ozean, wo sich die einzelnen Wirbel infolge der geringen Reibung (im Vergleich zum Festland, speziell Gebirgsgegenden!) gut entwickeln und lange halten können: Islandtief. Zwischen Hoch und Tief liegt die Grenzzone Kaltluft-Warmluft, die sogenannte Polarfront. An dieser entwickeln sich Randwirbel (Sekundärdepressionen), die mit der allgemeinen Strömung nach Osten wandern. Diese Randwirbel haben am Anfang ihrer Entwicklung einen sogenannten Warmsektor: die Warmluft südlich der Polarfront stößt an einzelnen 
Stellen gegen Norden vor (im Beispiel vom 4. 2. 1946 nordwestlich von Frankreich). Im weiteren Verlauf der Entwicklung wird aber dieser "Warmsektor» wieder kleiner. Die Warmfront auf der Vorderseite des Warmsektors, die auf die davorliegende Kaltluft aufgleitet, kommt in Bodennähe weniger rasch voran als die Kaltfront auf der Hinterseite dieses Gebietes. Die Warmluft hat in den unteren Schichten infolge ihrer geringen Dichte keine Stoßkraft. Die Kaltfront holt also die Warmfront ein: es bildet sich eine Okklusion (im Beispiel vom 4. 2. 1946 über Polen-Südskandinavien). - Der Durchgang der Fronten bringt in den betroffenen Gegenden starke Bewölkung und Niederschläge (aufsteigende Luftbewegung, die im Tief ja allgemein herrscht, aber besonders in der Gegend der Fronten stark ist).

Die Alpennordseite wird bei Westwindwetter im allgemeinen stärker von den Störungen berührt als der Alpensüdfuß, der im «Lee» der Alpen liegt. Die Schweiz befindet sich abwechslungsweise nördlich und südlich der Polarfront, also zeitweise in der Warmluft, zeitweise in der Kaltluft, wie die Temperaturtabelle der vier ausgewählten Stationen vom 3.-5. 2. 1946 zeigt $^{2}$. Entsprechend den starken Druckgegensätzen des Winterhalbjahres ist die Verlagerung der Störungen und der dazwischenliegenden Aufhellungen eine sehr rasche. Das Wetter hat bei Westwindlage typisch veränderlichen Charakter. Entsprechend der ausgleichenden Wirkung des Ozeans auf die darüber hinwegstreichenden Luftmassen ist es im Durchschnitt im Winterhalbjahr zu warm, im Sommer zu kühl bei Westwindwetterlage.

3. Die Wetterkarte vom 18.4. 1939 zeigt eine sogenannte Staulage, verursacht durch eine kühle Nordwest- bis Nordströmung gegen die Alpen. Wir sehen aus der Druckverteilung, daß die Staulage eine Föhnlage mit umgekehrtem Vorzeichen darstellt. Da wo beim Föhn Tiefdruck herrscht, ist hier ein Hoch und umgekehrt. Entsprechend sind auch die Wetterverhältnisse beidseits der Alpen vertauscht, beim Föhn Niederschläge südlich der Alpen, beim Stau auf der Alpennordseite, besonders in den östlichen .Voralpen (vgl. erhöhte Niederschlagssummen der Talstationen in diesem Gebiet auf der Regenkarte, S. 25 im Atlas). Auf der Alpensüdseite herrscht dagegen bei Staulage heiteres Wetter infolge absteigender Luftbewegung, des sogenannten Nordföhns (analog dem Südföhn der nördlichen Alpentäler). Infolge der ursprünglich kalten Luft ist jedoch der Nordföhn nicht so warm wie der Südföhn, aber ebenfalls sehr trocken und böig. Die von Norden her sich an den Alpen stauende Luft fließt nicht nur mit der Nordföhnströmung darüber hinweg, ein Teil davon sucht sich den Weg mit Westwind von der Ostschweiz nach Bayern-Österreich, ein anderer Teil aber drängt sich durch das untere Rhonetal nach dem Mittelmeer, wobei im relativ engen Tal sehr große Windgeschwindigkeiten auftreten: der sogenannte Mistral (vgl. im Beispiel Marseille $60 \mathrm{~km} / \mathrm{h}$.).

Die Staulage entsteht normalerweise beim Abschluß einer Zyklonenfamilie, wenn die Kette von Tiefdruckgebieten, die wir im zweiten Beispiel bei der Westwindwetterlage sahen, abbricht. Dann stößt die nördliche Kaltluft weit nach Süden vor. Es bildet sich eine Hochdruckbrücke zwischen dem Polarhoch und dem Azorenhoch. Die Vorbedingungen der Entstehung solcher Hochdruckkerne in den gemäßigten Breiten sind leider noch wenig geklärt. Offenbar ist dazu nicht nur ein Kaltluftausbruch nach Süden, sondern zugleich in entsprechendem Abstand westlich davon ein Warmluftvorstoß nach Norden (im Beispiel links außen) für den Aufbau und den Bestand des Hochs notwendig. In unserem Beispiel sehen wir rechts das letzte Tief der Zyklonenserie, hinter dem die Kaltluft nach Süden strömt. Wenn das Hochdruckgebiet auf dem Ozean ziemlich westlich liegt (z. B. westlich von Irland), ferner das Tief im Osten kräftig ent-

${ }^{2}$ Leider hat beim Westwindwetter der Druckfehlerteufel eifrig gewütet. Die Temperatur auf dem Säntis ist am 4. 2.07 Uhr von $3^{\circ}$ auf $-3^{\circ}$, diejenige auf dem Jungfraujoch von $9^{\circ}$ auf $-9^{\circ} \mathrm{zu}$ korrigieren. Beim Wind von Zürich ist WSW anstatt NSW zu lesen. (Diese und andere Fehler sind durch ein Versehen bei der Redaktion - also nicht des Autors - stehengeblieben. E. I.) 
wickelt ist und sich (infolge weitgehender Okklusion) nur noch wenig verlagert, kann die Kaltluftzufuhr in breitem Strom mehrere Tage anhalten. Solange zugleich Staulage herrscht, ist der Himmel auf der Alpennordseite bedeckt, bei nachfolgender Aufheiterung kann jedoch die kräftige Abkühlung im Frühjahr zu Nachtfrostgefahr führen.

Im normalen Ablauf einer Staulage entfernt sich das die Zyklonenserie abschließende Tief allmählich nach Osten. Das ozeanische Hoch dehnt sich nach Mitteleuropa aus, wodurch die Staulage in eine Bisenlage übergeht. In einzelnen Fällen wird das Hoch schon so rasch wieder durch eine neue Tiefdruckserie von Westen her abgebaut, daß keine Winddrehung nach Nordosten erfolgen kann, sondern die Staulage, die eine Westwindwetterperiode abschloß, direkt wieder in eine neue solche Periode überleitet.

4. Zur Entstehung einer Bisenlage ist hoher Druck über Mitteleuropa, tiefer Druck im Mittelmeerraum notwendig. Diese Luftdruckverteilung stellt sich ein, wenn ein starker Kaltluftvorstoß von Osten, Norden oder Nordwesten die Warmluft weit nach Süden zuirückdrängt. Dann liegt die Polarfront mit den sich an ihr bildenden Tiefdruckzentren (Depressionen) im Mittelmeergebiet. In der nördlichen Kaltluft ist der Luftdruck hoch; hat die Luft doch infolge der tiefen Temperatur eine große Dichte (vgl. Klimakarten: Sibirienhoch im Winter). Als Kaltluftquelle kommt für unsere Gegenden neben dem Polargebiet und Grönland vor allem das winterliche Rußland in Betracht. Unser Beispiel zeigt ein solches Ausfließen der russischen Kaltluft nach Westen und Südwesten, wobei in der Schweiz sehr starke Bise, an der dalmatinischen Küste ein dem Nordföhn verwandter Fallwind, die sog. Bora, weht (vgl. Windangaben der Stationen im Beispiel vom 23. 1. 1933). Wie wir aus diesem Beispiel ersehen, ist die Bise am stärksten in den unteren Luftschichten (meist zwischen 1000 und $1500 \mathrm{~m}$ ). Darüber nimmt die Windstärke ab. Wir können dies verstehen, wenn wir uns die Veränderungen der Luftdruckverteilung in den höheren Luftschichten vorstellen. In der schweren Kaltluft nimmt der Luftdruck mit zunehmender Meereshöhe rasch, in der warmen, leichten Luft des Mittelmeertiefs dagegen nur langsam ab. Also im russischeuropäischen Hoch rasche, im Mittelmeergebiet langsame Druckabnahme mit der Höhe. Die Luftdruckunterschiede sind in den höheren Luftschichten schwächer als am Boden; die Windstärke nimmt nach oben ab. In extremen Fällen finden wir sogar über der mitteleuropäischen Kaltluft ein Tief oberhalb $3000-5000$ m, d. h. das Mittelmeertief verlagert sich mit zunehmender Höhe immer mehr nach Norden. Über der südlichen Warmluft dagegen finden wir in diesen Schichten infolge der langsamen Druckabnahme nach oben ein (schwaches) Hoch. Also Tief im Norden, Hoch im Süden, d. h. Westwind, während unten kräftige Bise weht. Wir sehen, wieso der Meteorologe nicht nur die Wetterkarte am Boden (auf Meeresniveau), sondern auch als Ergänzung Höhen-Wetterkarten für die genaue Analyse der Wetterlage zeichnet.

Die mit der Bise heranströmende Kaltluft, die sich in den klaren Winternächten über den Schnee- und Eisflächen Nord- und Osteuropas abgekühlt hatte, stößt infolge ihrer Schwere längs dem Erdboden vor. Die Kaltluftschicht hat eine gewisse Mächtigkeit, die beim allmählichen Auseinanderfließen nach Westen immer mehr abnimmt. In unseren Gegenden ist die Kaltluft oft nur noch 1500-2000 m hoch, bei starken Vorstößer von Rußland her, wie im Beispiel, reicht sie bis zirka $3000-3500 \mathrm{~m}$. An der Obergrenze dieser Schicht bildet sich eine Temperaturinversion (Zunahme der Temperatur mit der Höhe statt der normalen Temperaturabnahme; vgl. Beobachtungen von Säntis und Jungfraujoch). Ferner entsteht unter der Inversionsschicht oft eine Hochnebeldecke, während darüber der Himmel heiter ist.

Im Sommer wirkt sich die Bisenlage etwas anders im Witterungscharakter aus als in der kalten Jahreszeit. Dann ist Rußland verhältnismäßig warm. Das charakteristische Merkmal der Luftströmung aus dem Osten ist dann die Trockenheit. Bise im Sommer bringt daher heiteres, ziemlich warmes Wetter. Da die Luftdruckunterschiede im Sommer geringer und die Vorbedingungen für die Entstehung eines Hochdruckgebietes 
auf dem Festland ungünstiger sind, tritt die Bise im Sommer schwächer und seltener auf; meist finden wir in der warmen Jahreszeit den Wechsel zwischen windschwachen Wetterlagen (vgl. nachfolgend Beispiele 5 und 6) und dem Westwindwetter.

5. Gewitterlage: Die Gewitter entstehen, wie wir aus der neueren Erforschung der freien Atmosphäre wissen, durch Auslösung der sogenannten Feuchtlabilität, die wir in ein paar Sätzen nachfolgend kurz skizzieren möchten:

Wenn die bodennahe Luftschicht über den Landfächen im Frühling und Sommer stark erhitzt wird, die oberen Luftschichten aber relativ kühl sind, ist die Temperaturabnahme mit der Höhe sehr groß. Sie erreicht in diesen Fällen Werte von $1^{\circ}$ pro $100 \mathrm{~m}$, wie wir es vom absteigenden Teil der Föhnströmung kennen, während ja normalerweise der Temperaturgradient nur zirka $1 / 2^{\circ}$ pro $100 \mathrm{~m}$ beträgt (vgl. Temperaturbeobachtungen im Beispiel vom 7. 7. 1936, wo die Temperaturabnahme von Basel $[300 \mathrm{~m}]$ über Rigi [1800] nach dem Jungfraujoch [3500 m] $0,9^{\circ}$ pro $100 \mathrm{~m}$ beträgt, an einzelnen überhitzten Stellen also $1^{\circ}$ pro $100 \mathrm{~m}$ sicher erreicht wird). Ein Luftteilchen, das am Erdboden stark erhitzt wird, steigt in die Höhe (Flimmern der Luft über Asphaltstraßen und Eisenbahngeleisen). Dabei kühlt es sich, solange keine Kondensation eintritt; also außerhalb der Wolken, um $1^{\circ}$ pro $100 \mathrm{~m}$ ab, in den Wolken nur noch um $1 / 2{ }^{\circ}$ bis $34^{\circ}$ pro $100 \mathrm{~m}$. Falls nun, wie in unserem Fall, der Temperaturgradient in der Atmosphäre nahe an $1^{\circ}$ pro $100 \mathrm{~m}$ herankommt, wird das aufsteigende Teilchen nicht wie in normalen Fällen beim Aufsteigen nach kurzer Zeit kälter und schwerer als seine Umgebung. Es kann, besonders von der Höhe weg, wo es kondensiert (Wolken bildet), sogar wesentlich wärmer und daher leichter als seine Umgebung bleiben. Es bilden sich ganze Luftpakete, die aufsteigen und dabè durch die blumenkohlförmigen Haufenwolken (Cumulus) sichtbar werden. (Segelflug!) Bei starker Entwicklung der Labilität, d. h. wenn die Luft der Atmosphäre wesentlich kälter ist als die aufsteigende Luft, können sich die Haufenwolken so kräftig entwickeln, daß sie in Gewitterwolken(Cumulonimbus) übergehen. Diese Entwicklung setzt an den besonders stark erhitzten wald- und schneearmen Zonen der höheren Voralpen beidseits der Alpen und der Juragipfel an vielen Sommertagen ein, wenn zwei wesentliche Vorbedingungen erfüllt sind: 1. Die Station darf sich nicht im zentralen Teil einer Antizyklone befinden; denn in diesem hemmt die absteigende Luftbewegung die Wolkenentwicklung (siehe nachfolgendes Beispiel 6). 2. Es darf keine stärkere Windströmung herrschen, wie sie in aktiven Depressionen auftritt, da sonst die lokal überhitzten, aufsteigenden "Luftpakete» zu stark auseinandergerissen werden. Voraussetzung für die Entstehung dieser lokalen, sogenannten Wärmegewitter ist also eine flache Druckverteilung bei mittlerem Barometerstand. (Wärmegewitter im Beispiel: Locarno 7..7.1936 nachmittags.)

Eine andere Klasse stellen die sogenannten Frontgewitter dar, die nicht wie die Wärmegewitter lokal an einzelnen Orten nachmittags oder abends auftreten, sondern in ganzen Gewitterzügen breite Landstriche überqueren. Diese Gewitter entstehen nicht an einzelnen, besonders für die Gewitterbildung günstigen Orten innerhalb der Warmluft, sondern an der Grenze zwischen warmer (kontinentaler) und kühlerer (maritimer) Meeresluft. Sie bilden sich auf breiter Front und wandern über große Distanzen. Man trifft sie beinahe zu allen Tageszeiten, allerdings selten am Morgen. Bei stärkerem Vorstoß der Kaltluftmassen nach Osten sind diese Gewitter meist von "schlechtem» Wetter gefolgt, während es hinter den Wärmegewittern wieder aufhellt. Die Entstehung der. Frontgewitter ist darauf zurückzuführen, daß bei den Wetterlagen mit flacher Druckverteilung am Boden die Windgeschwindigkeit in der Höhe im Grenzgebiet zwischen warmer und kalter Luft rasch zunimmt. (Den Grund dafür, die verschieden rasche Luftdruckabnahme mit der Höhe in der Kaltluft und der Warmluft haben wir bei der Bisenlage schon besprochen.) Infolge der großen Windgeschwindigkeit in der Höhe stößt die Kaltluft nicht wie im Winter längs des Bodens vor, sondern vor allem in den höheren Luftschichten. Dies bewirkt eine zunehmende Vergröß'erung des 
Temperaturgradienten, eine Labilisierung, deren Folgen wir oben besprochen haben: rasch aufsteigende Luftmassen vom Erdboden her, Ersatz dieser Luft aus der Höhe, also plötzlicher Umsturz der Luftmasse mit Gewittern im Umlagerungsgebiet ${ }^{3}$ (vgl. Durchgang der Gewitterkaltfront im Beispiel in der Schweiz von Westen nach Osten am Abend und in der Nacht des 7.7.1936). Während wir es also bei den Wärmegewittern mit rein vertikalen Umlagerungen, mit reiner Konvektion zu tun haben, wird bei den Frontgewittern die Konvektion durch eine leichte Advektion, durch eine schwache Kaltluftzufuhr ausgelöst. Dabei spielt aber energiemäßig die Advektion eine geringe Rolle; sie ist gewissermaßen nur Katalysator, der die großen Energien zur Auslösung bringt, die die Sonne mit ihrer gewaltigen "Heizung» tagaus, tagein dem Erdball liefert.

Alle Wetterlagen, alle Hoch- und Tiefdruckgebiete beziehen ihre Energie schließlich von der Sonne. Sie leben von den Gegensätzen in der Einstrahlung und Ausstrahlung zwischen Land, Meer und Luft und versuchen, diese Gegensätze auszugleichen. Wir sahen bei den Advektionswetterlagen, wie dieser Ausgleich im Winter vorwiegend durch horizontalen Lufttransport geschieht, während wir bei der Gewitterlage den sommerlichen Typus des Ausgleichs in vertikaler Richtung vor uns haben.

6. Schönwetterlage und Hochdruck sind in unserem Bewußtsein meist eng miteinander verknüpft, sehen wir diese Verbindung doch täglich an jedem Barometer angeschrieben. Und diese Kopplung ist (im Gegensatz zu den wohl ebenso verwurzelten Mondregeln!) auch wirklich vorhanden, wenn sie auch nicht ganz so einfach und allgemein gültig ist, wie dies die Barometerbeschriftung haben möchte. Die absolute Höhe des Barometerstandes ist nämlich weniger wichtig als der Luftdruckunterschied gegenüber den Nachbargebieten. Unser Land muß im zentralen Teil eines Hochdrucks liegen, um schönes Wetter zu erhalten. Es ist leicht ersichtlich, daß die Wahrscheinlichkeit für das Eintreffen dieser Vorbedingung um so größer ist, je höher das Barometer steht.

Aus dem Hoch fließt die Luft allseitig in die umgebenden Tiefdruckgebiete ab, allerdings nicht radial, sondern mit der durch die ablenkende Kraft der Erdrotation verursachten Rechtsdrehung (auf der Nordhalbkugel). Die Luft, die ausfließt, wird im zentralen Teil des Hochs durch Absinken der Luft aus der Höhe und entsprechenden Zufluß in den oberen Atmosphärenschichten ersetzt. Dabei erwärmt sich die absinkende Luft um $1^{\circ}$ pro $100 \mathrm{~m}$, also sehr stark im Verhältnis zum normalen Temperaturgradienten von $1 / 2^{\circ}$ pro $100 \mathrm{~m}$. Entsprechend nimmt die relative Feuchtigkeit ab. Die Luft wird warm und trocken. Im Winter kann sich zwar am Boden durch die Ausstrahlung in den langen Nächten eine Bodenkaltluftschicht halten, in der vielfach Nebel oder tiefer Hochnebel herrscht; darüber aber ist es warm (Temperaturinversion). Da das. Wetter wolkenlos oder nur leicht bewölkt ist, wird die tägliche Temperaturschwankung im Sommerhalbjahr im Flachland und in den Alpentälern sehr groß, während sie auf den Berggipfeln und in der freien Atmosphäre klein ist (vgl. z. B. die in gleicher Meereshöhe gelegenen Orte Rigi und St. Moritz im Beispiel des 29. 8. 1936). Die relativen Feuchtigkeiten und die Temperaturen zeigen im Tal starke Schwankungen. Auf den Bergen ist die Feuchtigkeit besonders am Vormittag klein, entsprechend der oben besprochenen Absinkbewegung. Beim Rigi und Säntis macht sich im Beispiel die mittägliche Erhitzung der umliegenden Talsohlen und die dadurch verursachte Instabilität durch aufsteigende Luftbewegung längs der Berghänge bemerkbar, daher die Feuchtigkeitszunahme am Mittag. Es bilden sich dann Schönwetterwolken über den Gipfeln. «Hat der Pilatus einen Hut, so ist das Wetter gut.»

s Im Beispiel vom 7. 7. 1936 ist die Grenze zwischen Warm- und Kaltluft für die höheren Luftschichten in Belgien und Frankreich durch eine (Höhen-)Kaltfrontlinie eingezeichnet. Sie ist gegenüber der Grenzlinie am Boden, die ungefähr mit der $20^{\circ}$-Isotherme zusammenfällt, nach Osten vorgeschoben. Charakteristisch ist dabei die Bildung einer Tiefdruckfurche, eines "Gewittersacks» über Ostfrankreich. 
Wir sehen bei der Schönwetterlage sehr klar den Einfluß der Jahreszeiten auf die Bewölkung: im Sommer Bildung von Haufenwolken, besonders im Gebirge, wenig Wolken im Flachland; im Winter Bildung von Nebel und Hochnebel in der Ebene, geringe Bewölkung im Alpengebiet.

Zum Abschluß unserer Beschreibung wollen wir einen kurzen Blick aufs Ganze werfen, damit nicht die Gefahr besteht, daß wir vor lauter Bäumen den Wald nicht mehr sehen. Wir wollen ein kleines meteorologisches Jahrbuch aufstellen, nicht für ein Einzeljahr, keinen hundertjährigen Kalender, sondern eine Beschreibung des hauptsächlichsten Auftretens der einzelnen Wetterlagen. Wir können uns dabei vielfach volkstümlicher Bezeichnungen bedienen; sind doch die wesentlichsten Merkmale dieses Wetterablaufes in das Sprachgut eines großen Teiles des Volkes übergegangen.

Im Winter finden wir den Kampf der drei großen, in unserer Gegend wirksamen Aktionszentren: Rußlandhoch, Azorenhoch und Islandtief (vgl. Klimakarten S. 133: Luftdruckverteilung im Januar). Dabei verfrachtet das Rußlandhoch (wenn es genügend weit nördlich und westlich liegt) auf seiner Südseite die Kaltluft Osteuropas nach Westen. Azorenhoch und Islandtief dagegen befördern zusammen die ozeanische Warmluft nach Osten. Unser Gebiet steht abwechselnd unter dem Einfluß der beiden Strömungen und hat zeitweise Bise, zeitweise Westwind, dazwischen als Übergang Staulagen und einzelne Föhnlagen. In milden Wintern überwiegt die westliche, in strengen die östliche Luftzufuhr. Dann folgt gegen das Winterende eine ruhigere Periode. Die antizyklonalen Schönwetterlagen, die im Hochwinter meist nur kurzen Bestand haben, werden häufiger und beständiger, besonders in der zweiten Hälfte Februar und um Mitte März. Wir sind im «neutralen» Gebiet.

Gegen den Sommer schwächt sich das russische Hoch ab, da sich der Kontinent erwärmt. Die ozeanische Westwindströmung dringt gegen Mitteleuropa vor, kann sich aber hier noch nicht richtig durchsetzen, sondern biegt in Westeuropa nach Norden um. Wir sind in der Zeit der Föhnlagen, der Schneeschmelze. Dann dringt der Westwind weiter vor: Aprilwetter. Im Mai flaut die Zufuhr von Westen vorübergehend ab. Die winterliche Energie der beiden Aktionszentren Azoren-Island ist aufgebraucht; der sommerliche Antrieb, der durch den Temperaturgegensatz Meer-Festland gebildet wird, ist noch nicht voll in Betrieb. Wir befinden uns in der vorsommerlichen Schönwetterlage von Mitte Mai.

Dazwischen kommen einzelne letzte Kaltlufteinbrüche aus Norden, sehr unregelmäßig, nicht nur an den Eisheiligen, ja dann sogar im meteorologisch erforschten Zeitraum der letzten hundert Jahre nicht einmal besonders häufig. Diese Erscheinung der Kälterückfälle zur Zeit der Eisheiligen war offenbar eine kalendermäßige Bindung, eine sogenannte Singularität früherer Jahrhunderte, die durch die Überlieferung weiterbesteht und sicher noch lange geglaubt werden wird, unbekümmert um alle Statistiken, die keinen solchen Einfluß mehr erkennen lassen. Auch die Mondregeln werden bekanntlich weiterbestehen, ob sie stimmen oder nicht...

H Dann beginnt der Sommer: Überhitzung des Kontinentes, während es in der Höhe anfänglich noch kalt ist (die Sonne heizt ja nicht die Luft, sondern den Erdboden!): Gewitterlagen, besonders im Mai, Juni und Juli. Die über den großen Landflächen erwärmte Luft dehnt sich nach der Höhe aus, ein Teil strömt nach den Meeresgebieten ab, so daß der Luftdruck über dem Festland fällt (vgl. Klimakarten S. 133: Luftdruckverteilung im Juli). In das am Boden entstehende Tief brechen nach einiger Zeit die kühlen und feuchten ozeanischen Luftmassen ein und lösen Frontgewitter aus. Besonders häufig ist dieser Vorgang um Mitte Juni, so daß um diese Zeit sogar die langjährige Temperatur-Jahreskurve eine Abkühlung zeigt. Dies ist die bestausgeprägte Singularität in unserer Zeitepoche.

In der zweiten Hälfte des Sommers sind auch die höheren Atmosphärenschichten durch die vorangegangene Gewittertätigkeit und die damit verbundenen Umlagerun- 
gen stärker durchwärmt, während sich in den unteren Schichten schon die beginnende herbstliche Abkühlung bemerkbar macht. Dann werden die Vorbedingungen für die Gewitterlagen seltener, und wir finden häufig Schönwetterlagen, anfangs mit großer Hitze, in den "Hundstagen», später mit mäßiger Erwärmung im "Altweibersommer». Die Witterung wird «beständig», wobei dieser Ausdruck nicht nur für das heitere, sondern auch für das regnerische Wetter gilt. Wenn es sich einmal "eingelassen hat» um die Pfingstzeit, sind die Aussichten für schönes Sommerwetter bekanntlich nicht sehr günstig. Viele der zum Teil miteinander, zum Teil gegeneinander arbeitenden Ursachen für die Witterungsgestaltung ganzer Jahreszeiten sind noch in Dunkel gehüllt, daher ist ja auch eine Wetterprognose auf längere Zeiträume bisher unmöglich.

Auch der Herbst weist häufig ruhige, antizyklonale Wetterlagen auf, doch beginnt die Westwindströmung auf dem Ozean schon wieder an Stärke zuzunehmen. Sie verursacht eine zweite Föhnzeit, mit stärkster Entwicklung meist gegen Ende Oktober: Dazwischen kommen die ersten Kaltluftausbrüche aus dem Polarraum mit Staulagen. Dann setzt sich die Westwindströmung wieder bis nach Mitteleuropa durch: Novemberstürme. Jetzt erst wird das Rußlandhoch über den gebildeten Schneeflächen allmählich stark. Wir gelangen im Dezember wieder ins neutrale Grenzgebiet mit antizyklonalen Wetterlagen, von denen es im Wetterbericht heißt: Niederungen Hochnebel, Höhen heiter. Um die Weihnachtszeit beginnt dann normalerweise der anfangs besprochene Kampf zwischen Ost- und Westströmung, oft mit Tauwetter als Auftakt. Unser Jahreskreislauf ist geschlossen.

Wir sind uns bewußt, mit unseren sechs Grundtypen keine erschöpfende Darstellung der Wetterlagen geben zu können. In hundert Jahren gibt es $100 \times 365$ Tageswetterkarten; keine gleicht genau der andern. Aufgabe des Betrachters ist es (mit Hilfe des erklärenden Textes des Meteorologen) eine Verbindung dieses Wetterkartenbildes mit dem beobachteten Wetter herzustellen, eine Erklärung und Einordnung der Beobachtungen in ein festes Schema und damit die Grundlagen für eine eigene Beurteilung der Wetterlage zu gewinnen. Möchten recht viele dabei angeregt werden, selbst aufmerksam das Wetter zu beobachten und die Schönheit der Wolken- und Wetterentwicklung in sich aufzunehmen.

\title{
RÉSUMÉ CONCERNANT LES LÉGENDES DES TYPES DE TEMPS DE L'ATLAS POUR L'ENSEIGNEMENT SECONDAIRE
}

\begin{abstract}
A la place des seules cartes représentant les conditions de formation du fohn, le nouvel atlas contient des exemples caractéristiques des six types de temps fondamentaux qui permettent une étude plus complète de la météorologie. Il s'agit de quatre types de temps caractérisés par un déplacement d'air horizontal: temps de fœhn, de vent d'ouest, de vent du nord et de régime de bise, puis de deux autres types avec prédominance de mouvement vertical: le temps d'orage et le beau temps. Un choix de bulletins météorologiques permet de se rendre compte des conditions atmosphériques correspondant aux différentes cartes. Dans le présent article, on expose en détail les points essentiels qui interviennent dans le commentaire et l'appréciation de ces types caractéristiques. On traite, d'autre part, de la fréquence saisonnière des types de temps particuliers.
\end{abstract}

\section{RIASSUNTO DELLE SPIEGAZIONI INERENTI ALLE SITUAZIONI METEORO- LOGICHE TIPICHE RAPPRESENTATE NELL'ATLANTE PER LE SCUOLE MEDIE}

Invece dell'unica carta del favonio esistente finora, nella nuova edizione dell'Atlante sono contenute le rappresentazioni delle sei tipiche e fondamentali situazioni meteorologiche che permetteranno una trattazione più completa della meteorologia. Si tratta delle quattro situazioni di advezione con movimenti delle masse d'aria in prevalenza orizzontali e cioè : la situazione favonica, quella dei venti atlantici, la situazione dei venti dal nord e quella della bisa; inoltre i due tipi di convezione con movimenti prevalentemente verticali, dunque la situazione temporalesca e quella di bel tempo. Una selezione di segni convenzionali rappresentanti le particolarita fenomenologiche locali illustrano le variazioni orizzontali del tempo nell'ambito della stessa situazione tipica per la nostra regione. Nel presente lavoro sono spiegati quei concetti fondamentali della meteorologia che permettono di discutere e di válorizzare le singole situazioni. E da ultimo viene discussa la frequenza annuale dell'avverarsi delle singole situazioni tipiche. 